\title{
Identify the Centerline of Tubular Structure in Medical Images
}

\author{
Yu-Tai Ching ${ }^{a}$ and Carlos Yeh $^{a}$ \\ ${ }^{a}$ Department of Computer and Information Science, National Chiao Tung University, \\ HsunChu, Taiwan.
}

\begin{abstract}
Finding the centerline of the tubular structure helps to segment or analyze the organs such as the vessels or neuron fibers in medical images. This paper described a semi-automatic method using the minimum cost path finding and Hessian matrix analysis in scale space to calculate the centerline of tubular structure organs. Unlike previous approaches, exhaustive search for line-like shapes in every scale is prevent. Centerline pixels candidates and the width of the vessel are extracted by analyzing the intensity profile along the gradient vectors in the image. A verification procedure using Hassian matrix analysis with the scale obtained from the gradient analysis is applied to those candidates. Results obtained from the Hessian matrix analysis are used to construct a weighted graph. Finding the minimum cost path in the graph gives the centerline of the tubular structure. The method is applied to find the centerline of the vessels in the $2 \mathrm{D}$ angiogram and the neuron fibers in the $3 \mathrm{D}$ confocal microscopic images.
\end{abstract}

Keywords: Hessian matrix analysis, centerline, graph algorithm, angiogram, confocal microscopic image.

Many organs, such as blood vessel and neuron, inside human body are tubular shaped. knowing the centerline of these organs help to obtain quantitative information, such as shape and size of the organ, for further analysis. In this paper, we present a semi-automatic method to extract the centerline of the tubular structure in 2D and $3 \mathrm{D}$ images. The method was applied to segment the $2 \mathrm{D}$ vessel in angiogram and the $3 \mathrm{D}$ neuron in the confocal microscopic images.

Centerline of a tubular structure can be obtained by first segment the region of interest to establish a binary image of the ROI. A further procedure can then be applied to calculate the centerline. A problem with this approach is that the ROI could be difficult to segment. For example, segmentation of the coronary arteries in the angiogram is a difficult problem.

Coronary arteries angiography is still the most common modality for physicians to assess the severity of coronary narrowing or stenosis during percutaneous coronary intervention procedure. Accurate analysis of coronary arteries in digital angiographic images is valuable and important to clinical needs. The major difficulty in automatic extraction of coronary arterial structures in angiogram lies in (1) low signal-to-noise ratio due to poor X-ray penetration, (2) vessel overlaps, and (3) superimposition of other tissues such as ribs, spine, or cardiac chambers. Traditional signal-based edge detection algorithms ${ }^{1-7}$ were unable to effectively or accurately detect the desired structures. The existing methods specific to vessel extraction can be categorized into (i) model-based ${ }^{8-10}$ (ii) tracking-based, ${ }^{11-13}$ (iii) classifier-based, ${ }^{14}$ and (iv) filter-based ${ }^{15-17}$ techniques. In model-based methods, the coronary arterial tree is produced based on a pre-defined coronary artery model in the form of a "graph" structure. In tracking-based methods, the process proceeds with an initial start-of-search location followed by an automatic tracking process by exploiting the spatial continuity of the vessel's centerline, orientation, diameter, and density. In classifier-based methods, a clustering algorithm is employed with properly preprocessed data to differentiate vessel or non-vessel regions. In filter-based methods, the coronary arteries are enhanced and located so that they can be subsequently detected in the image. However, any of the above approaches cannot guarantee to work well in all of the cases.

\footnotetext{
Further author information: (Send correspondence to Yu-Tai Ching)

Yu-Tai Ching: E-mail: ytching@cis.nctu.edu.tw, Telephone: +886-3-5131547

Carlos Yeh: E-mail: carlos@cis.nctu.edu.tw
} 
Another case studied in this paper is tracing neuron fibers in the confocal microscopic images. A neuron consists of necleus, dendrite, axon, and terminal button. Knowing the centerline of the neuron helps to analyze the shape of the neuron. Due to the low contrast of the image and the shape of the neuron, segmentation of neuron fibers in confocal microscopic images is a much difficult task than segmentation of the vessels in angiogram. To calculate the centerline by first segmentation of the neuron fibers might not be an appropriate approach.

In this paper, we developed a method to calculate the centerline of tubular structure directly from the image. The technique is developed based on the Hessian matrix analysis in scale space. The Hessian matrix analysis evaluates the local curvatures to explore the second order structure surrounding at a point such that results of analysis is independent of image properties, and is only dependent to the local shape of the point. Hence, they generally are suitable for different types of images. Combined with the Hessian matrix analysis, multi-scale technique is used to detect every scale of feature and eliminates the effect of noise to construct a robust and feasible extraction algorithm. By finding the centerline directly from the image, we could develop an easier method to segment the tubular structure.

For completeness, we briefly state the background of the multi-scale technique. Lindeberg ${ }^{18-20}$ revealed the way to detect ridge lines in an 2D image for computer vision using analysis of local directional derivatives to find the area at which the shape conform the ridge definition in differential geometry. His algorithm calculates directional derivatives of every pixel in scale-space, which represents different level of detail, from the coarse to the fine, of the image. The directional derivatives of a pixel at some scale can be viewed as a kind of description of the local shape at that scale. Evaluation and selection those descriptions in scale-space results the possible ridge, or say, centerline of line structures. Sato et al. ${ }^{21}$ present a generalized measure of similarity of line in 3D space using calculation of eigenvalues of the Hessian matrix, which is equivalent to the directional derivatives. They designed a delicate formula to estimate the likelihood of being line-shape from eigenvalues of the Hessian matrix, and build a 3D line filter by it for vessel enhancement. Frangi et al. ${ }^{22}$ also propose another line filter based on analysis of the Hessian using their own formula. It is shown by the experimental result that good noise and background suppression of this method. A line extraction algorithm using the response of line filter mentioned above and the minimum cost path finding is proposed by Wink et al.. ${ }^{23}$ The response from the line filter is regarded as weights of edges in a graph where image pixels are viewed as nodes and there are edges connected between any two adjacent pixels in it. Hence, given two points as the beginning and ending of a vessel or neuron, finding a minimum cost path (with highest weight) extracts the most possible centerline of the vessel or neuron. Wink et al. improved their tracking algorithm by involving scale parameters into minimum cost path finding. ${ }^{24}$ In previous methods using minimum cost finding combined with the line filter to obtain centerlines, the response using as weights of graph is generally taken from the maximum response over the scales in scale-space. In this method, however, response in every scale is reserved as a part of the weights in path finding, rather than discarding all except the max one. This strategy improves the accuracy of the algorithm but need huge of memory. This restricts the application of the algorithm to the case of handling 3D volume data.

In this paper, we present a semi-automatic method to segment tubular structure in $2 \mathrm{D}$ image or 3D volume data. The method reduces the memory required so processing large volume in a personal computer is possible. In the next section, we describe some preliminaries. In Section 2, we present our method. The results are shown in Section 3. We have conclusion and discussion in Section 4

\section{PRELIMINARIES}

We briefly describe the 3D Hessian Matrix analysis. The 2D Hessian Matrix is the same but ignore the third dimension. Hessian matrix for a point $p$ under scale $\sigma$ in 3D space can be written as the following equation

$$
H_{\sigma}(p)=\left[\begin{array}{ccc}
I_{x x} & I_{x y} & I_{x z} \\
I_{y x} & I_{y y} & I_{y z} \\
I_{z x} & I_{z y} & I_{z z}
\end{array}\right],
$$


where $I_{a b}$ is the partial derivative of $I$ in $a, b$ direction $\partial^{2} I / \partial a \partial b$. If $I$ is continuous, $I_{a b}=I_{b a}, H$ can be written as a symmetric matrix

$$
H_{\sigma}(p)=\left[\begin{array}{ccc}
I_{x x} & I_{x y} & I_{x z} \\
I_{x y} & I_{y y} & I_{y z} \\
I_{x z} & I_{y z} & I_{z z}
\end{array}\right] .
$$

Let $\lambda_{1}, \lambda_{2}$, and $\lambda_{3}$ be the eigen values of $H_{\sigma}(p)$, and $\lambda_{1} \leq \lambda_{2} \leq \lambda_{3}$. If $(x, y, z)$ is on the centerline of a neuron fiber, we have $\left|\lambda_{1}\right| \approx 0$ and $\left|\lambda_{2}\right|$ and $\left|\lambda_{3}\right|$ are large. Furthermore, let $\mathbf{v}_{1}, \mathbf{v}_{2}$, and $\mathbf{v}_{3}$ are respectively the eigen vectors corresponding to the eigen values $\lambda_{1}, \lambda_{2}$, and $\lambda_{3}$. We have $\mathbf{v}_{1}$ points along the direction of the medial axis, and $\mathbf{v}_{2}$ and $\mathbf{v}_{3}$ are normal to the medial axis.

Computing the second partial derivative of the volume data with scale $\sigma=w$ is implemented in the frequency domain. Given a point $p$, the scale $\sigma=w$ defines a Gaussian function $g(p, \sigma)$ that the mean is at point $p$ with variance $\sigma$. To compute the second partial derivative of the volume data is equivalent to

1. Fourier transform the volume data into frequency domain.

2. Compute the second partial derivative of the Gaussian Function and transform it into the frequency domain.

3. Multiply the two results in the frequency domain.

4. Inverse transforming the frequency domain back to the spatial domain.

The computation of the second partial derivatives are shown in Eqs. (3-8)

$$
\begin{aligned}
& L N_{x x}(p)=\sigma^{2} I(p) * \frac{\partial^{2}}{\partial x \partial x} g(p, \sigma) \\
& L N_{y y}(p)=\sigma^{2} I(p) * \frac{\partial^{2}}{\partial y \partial y} g(p, \sigma) \\
& L N_{z z}(p)=\sigma^{2} I(p) * \frac{\partial^{2}}{\partial z \partial z} g(p, \sigma) \\
& L N_{x y}(p)=\sigma^{2} I(p) * \frac{\partial^{2}}{\partial x \partial y} g(p, \sigma) \\
& L N_{y z}(p)=\sigma^{2} I(p) * \frac{\partial^{2}}{\partial y \partial z} g(p, \sigma) \\
& L N_{z x}(p)=\sigma^{2} I(p) * \frac{\partial^{2}}{\partial z \partial x} g(p, \sigma)
\end{aligned}
$$

The Hessian Matrix with at point $p$ for scale $\sigma$, denoted $H_{\sigma}(p)$, is established using the following equation.

$$
H_{\sigma}(p)=\left[\begin{array}{lll}
L N_{x x}(p) & L N_{x y}(p) & L N_{x z}(p) \\
L N_{x y}(p) & L N_{y y}(p) & L N_{y z}(p) \\
L N_{x z}(p) & L N_{y z}(p) & L N_{z z}(p)
\end{array}\right] .
$$

\section{METHOD}

The method consists of three steps, the preprocessing step, Hessian Matrix analysis, and constructing a weighted graph and find the shortest path in the graph. Each of the steps is described in the following. 


\subsection{Preprocessing}

Gradient in an image is generally used to compute the edge in the image. Using the Sobel operation, we compute the gradients, $S_{x}, S_{y}, S_{z}$ along $x, y$, and $z$ directions at point $(i, j, k)$. Let $p=(i, j, k)$ be a point, $\left(S_{x}(p), S_{y}(p), S_{z}(p)\right)$ describes the direction that the intensity changes the most and $\left|S_{x}\right|+\left|S_{y}\right|+\left|S_{z}\right|$ gives the magnitudes that the intensity changes. If $p$ is a boundary point, $\left|S_{x}(p)\right|+\left|S_{y}(p)\right|+\left|S_{z}(p)\right|$ tends to be large and $\left(S_{x}(p), S_{y}(p), S_{z}(p)\right)$ approximately points to the center of the tubular structure. Based on this observation, we shall look at the points that have the magnitude of the gradient greater than a given threshold $T_{g}$. And along the direction of the gradient, we shall determine the approximate boundaries and the estimated center point of the tubular structure. The estimated boundary points and center points are presented as the triple $\langle p, c, q\rangle$ where $p$ and $q$ are the estimated boundary points and $c$ is the estimated center point.

The preprocessing step is different for finding centerline for vessel (2D case) and neuron (3D case). We separate the discussion in two parts.

\section{D Vessel Case}

The triple is obtained by analyzing the intensity distribution along the direction of the gradient vector. Let $p$ be an boundary point obtained from the Sobel filtering. Let $\mathbf{v}$ be a directed line that has one end point anchor at $p$ and emitting along the direction of the gradient. Along $\mathbf{v}$, the intensity distribution can be roughly divided into five intervals, namely, flat interval, upward interval, ridge interval, downward interval, and flat interval. The five intervals along $\mathbf{v}$ indicate that the ray shooting into the vessel, passing the center of the vessel on the ridge interval, and exiting the vessel. The entering point, a point on the ridge interval, and the exiting points are respectively the points $p, c$, and $q$. The algorithm to determine the five intervals and the triple is described in the following.

Along $\mathbf{v}$, we discretize the directed line to a set of points, $\left\{s_{i} \mid s_{i}=p+i \cdot \epsilon \cdot \mathbf{u}, i=0, \ldots, n\right\}$, where $\mathbf{u}$ is the unit vector along $\mathbf{v}$. The intensity of each of the sampled point $s_{i}$ is denoted $I\left(s_{i}\right)$. If $s_{i}$ does not have integer coordinates, $I\left(s_{i}\right)$ is obtained using the bilinear interpolation from the four neighboring points. Each pair of $I\left(s_{i}\right), I\left(s_{i+1}\right), i=1, \ldots, n-1$ defines a slope $m_{i}$

$$
m_{i}=\frac{I\left(s_{i+1}\right)-I_{s_{i}}}{\epsilon}
$$

Depending on the slope, we classify the intervals into four categories, namely, flat, upward, downward, and ridge. Given a threshold, $T_{\text {flat }}$, the $i$ th interval is

- flat, if $\left|m_{i}\right|<T_{\text {flat }}$,

- upward, if $m_{i} \geq T_{\text {flat }}$,

- downward, if $m_{i} \leq-T_{\text {flat }}$, and

- ridge, if $\left|m_{i}\right|<T_{\text {flat }}$, and this interval is between an upward interval and a downward interval.

With these four intervals, for a given point $p, c$ and $q$ are determine if they meet one of the following two conditions,

- $q$ is in a downward interval and $I(p)=I(q)$. In this case, the centerline of the neuron, $c$, is $(p+q) / 2$.

- $c$ is the center of the ridge interval or at the boundary of the upward and downward intervals (ridge is empty). In this case, $q$ locates at $c+|\vec{p}|$.

For each computed $p, q$, and $c$, we have a triple $\langle p, c, q>$ which is a possible cross section of a vessel and $c$ is a candidate for a point on the centerline. Note that, for each point $p$, there could be a pair of triples calculated. Figure 1 show the triples (cross section) found according to the rules stated above. According to the rules, we find the set possible candidates for the centerline. The best possible result shall be determined by the shortest path finding algorithm. The set of points $C=\{c \mid c$ is a candidate for a point on the centerline $\}$ will be used for creating the weighted graph. 

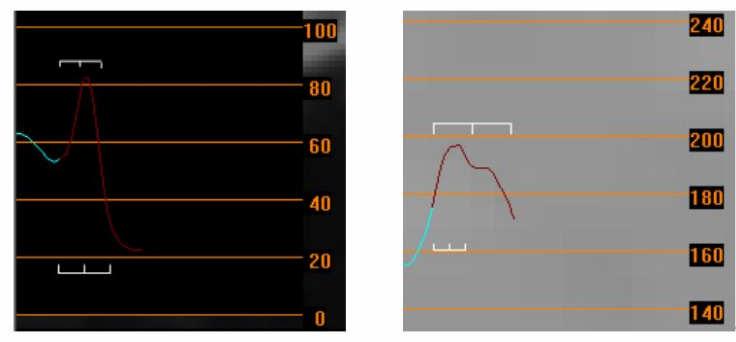

Figure 1. Two triples are obtained by applying the two rules. Each triple obtain a possible point on the centerline. The best one will be determined by the shortest path finding algorithm.

\section{D Neuron Case:}

When the gradient analysis is applied to $3 \mathrm{D}$ volume case, the steps performed are the same as that in the $2 \mathrm{D}$ case. Since the directed line determined by the gradient may not pass through the true center of a neuron fiber, the candidates for the points on the centerline are determined differently. Let $C^{\prime}$ be the set of points of all points $c$ found in gradient analysis and $C$ be the set of points obtained by applying the dilation operation of $C^{\prime}$. $C$ contains most of the points on the centerline.

\subsection{Hessian Matrix Analysis}

The points in $C$ is the set of candidate point for the centerline of the tubular structure. Note that the point in $C$ is obtained through the gradient analysis that the magnitude of the gradient is greater than a given threshold. Noises can also produce points in $C$. To verify whether a point in $C$ is truly on the centerline, we apply the Hessian Matrix analysis.

For each triple $\langle p, c, q\rangle$ in $C,|\overline{p, q}|$ is the estimated width of the vessel. Let $W=\overline{p, q} / 2$, we expect that there is a tubular structure with radius equal to the half width of the cross section, $W$, passing through $c$. By using a Hessain Matrix with scale $\sigma=W$, there should be a detected tubular structure.

2D Vessel Case

For each point $c$ in $C$, we compute the Hessian Matrix $H_{\sigma}(c)$.

$$
H_{\sigma}(c)=\left[\begin{array}{ll}
L N_{x x}(c) & L N_{x y}(c) \\
L N_{x y}(c) & L N_{y y}(c)
\end{array}\right] .
$$

Note that, the $\sigma$ used in obtained from the previous step. The two eigen values $\lambda_{1}$ and $\lambda_{2}\left(\lambda_{1}<\lambda_{2}\right)$ are calculated. We define $w_{1}$ and $w_{2}$,

$$
w_{1}=\frac{\left|\lambda_{1}\right|}{\left|\lambda_{2}\right|^{\frac{3}{2}}},
$$

and

$$
w_{2}=\frac{\left|\mathbf{v}_{\mathbf{2}} \cdot \overrightarrow{p, q}\right|}{\left|\mathbf{v}_{\mathbf{2}}\right||\overrightarrow{p, q}|},
$$

where $\mathbf{v}_{2}$ is the eigen vector corresponding to $\lambda_{2}$. Recall that, if $c$ is indeed on the centerline of the vessel of width $W,\left|\lambda_{1}\right| \approx 0$ and $\left|\lambda_{1}\right|<<\left|\lambda_{2}\right|$. That means $w_{1}$ should be very small. Secondly, if $\overline{p, q}$ is truly orthogonal to the centerline of the vessel, $\mathbf{v}_{2}$ should have same direction as $\overrightarrow{p,}$, , thus $w_{2}$ should be close to 1 . Combining $w_{1}$ and $w_{2}$, we define the weight $w$

$$
w=e^{\left(\frac{w_{1}^{2}}{-2 a^{2}}\right)} e^{\left(\frac{\left(1-w_{2}\right)^{2}}{-2 b^{2}}\right)} .
$$

$w$ is in the range $[0,1]$. If $w$ is large, there is significant line structure with width $W$ and $c$ is on the centerline.

$3 D$ Neuron Case

We compute the 3D Hessian Matrix as show in Eq. (9). Again, the three eigen values, $\lambda_{1}, \lambda_{2}$ and $\lambda_{3}$, are 
computed. Recall that if $c$ is indeed on the centerline of a tubular structure, we have $\lambda_{1}<<\lambda_{2}, \lambda_{1}<<\lambda_{3}$, and $\lambda_{2} \approx \lambda_{3}$. In this case, we define the weight $w$,

$$
w=\sqrt{\lambda_{2} \cdot \lambda_{3}}-\lambda_{1} .
$$

If $w$ is large then $c$ is likely on the centerline of a neuron fiber.

\subsection{Graph Construction and the Shortest Path Calculation}

Neuron structure extraction is carried out through finding the shortest path in a weighted graph $G=(V, E)$. Each vertex in $V$ corresponds to a point in $C$. Let $u, v$ be two vertices in $E$. There is an edge between $u$ and $v$ if the points corresponding to $u, v$ are 8-neighbor connected in 2D case or 26-neighbor connected in a 3D volume. There are costs associated with the edges in $E$. We used different way to define the weight of an edge.

$2 D$ Vessel Case

The weight between two connected vertices $u$ and $v$ is denoted $C(u, v)$.

$$
C(u, v)=\left(C_{\text {weight }}(i, j)+C_{\text {width }}(i, j)\right) \cdot C_{\text {distance }}(i, j) \cdot C_{\text {direction }}(i, j),
$$

where

$$
\begin{aligned}
C_{\text {weight }}(i, j) & =\frac{1}{w_{i}}+\frac{1}{w_{j}} \\
C_{\text {width }}(i, j) & =\left\lceil\left(\left|w_{i}-w_{j}\right|\right)+0.5\right\rceil \\
C_{\text {distance }}(i, j) & =|\overrightarrow{u, v}| \\
C_{\text {direction }}(i, j) & =\left(2-\frac{\left(1-\overrightarrow{v_{i}, v_{j}} \cdot d i r_{i}\right)^{2}}{2 c^{2}}\right)\left(2-\frac{\left(1-\overrightarrow{v_{i} v_{j}} \cdot d i r_{j}\right)^{2}}{2 c^{2}}\right)
\end{aligned}
$$

$3 D$ Neuron Case

For an edge $e$ connecting $u$ and $v$, we define

$$
C(u, v)=w_{1}+C_{\text {distance }}
$$

where $C_{\text {distance }}(u, v)=D\left(v_{i}, v_{j}\right)$.

The weights are designed so that the centerline of a piece of vessel or neuron fiber occur on the shortest path between a pair of vertices on the neuron. Given the weighted graph, $G=(V, E)$, we calculate the all pairs shortest paths using the Dijkstra Algorithm. The structure extraction algorithm is obtained by a graphics user interface system. The user provides a pair of points on the centerline as the input. The shortest path between the pair of points are calculated that corresponds to the centerline between the points. The process iterates until the whole tubular structure is extracted.

\section{RESULTS}

In Figure 2, a computer generated phantom test data is shown. The centerline obtained by the proposed method is shown on the right side. In order to test the robustness of the proposed method, we used computer to generate low contrast phantom image. We also generated the condition that the background intensity changes gradually (Figure 3). The image containing noises were also generated by computer for testing robustness reason(Figure 4). In Figure 3, since the background intensity changes, the centerline shift to the right of the true centerline. But the centerline falls in the interior of the vessel. That means the centerline can still serve as a reference to find the boundary of vessel. In the noisy image shown in Figure 4, the proposed method can still accurately calculate the centerline.

In Figure 5, the retinal images is shown. The centerlines for the vessels in the low contrast area could still be well identified. The experiments using the coronary artery angiogram was also performed (Figure 6). The arterial tree can be extracted fairly complete.

In Figure 7, we present the result obtained by tracing neuron in 3D confocal microscopic volume data. The image on the right was obtained by tracing neuron fibers in a volume data of size $512 \times 512 \times 120$. This problem can be fitted into a personnel computer with $2 \mathrm{G}$ memory. The preprocessing time took about two hours. 


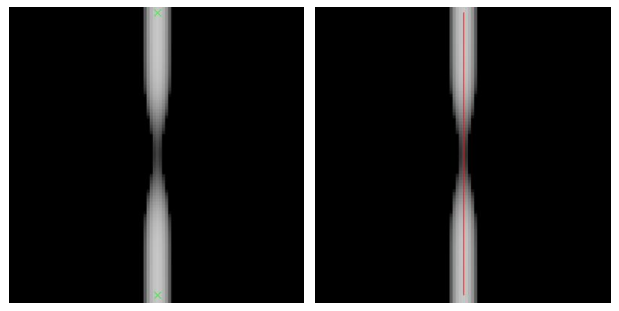

Figure 2. A computer generated phantom data set. The original image is shown on the left and the center line obtained is show on the right.

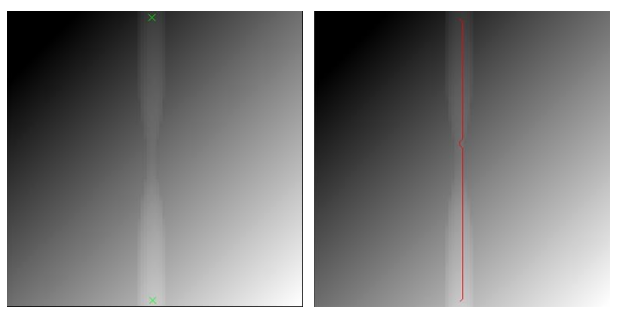

Figure 3. The test data containing low contrast image and the background intensity change gradually. The original image is shown on the left and the center line obtained is show on the right.

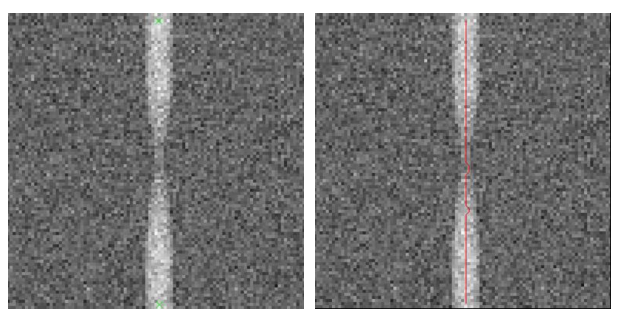

Figure 4. Image containing noises. The original image on the left and the result shown on the right. 

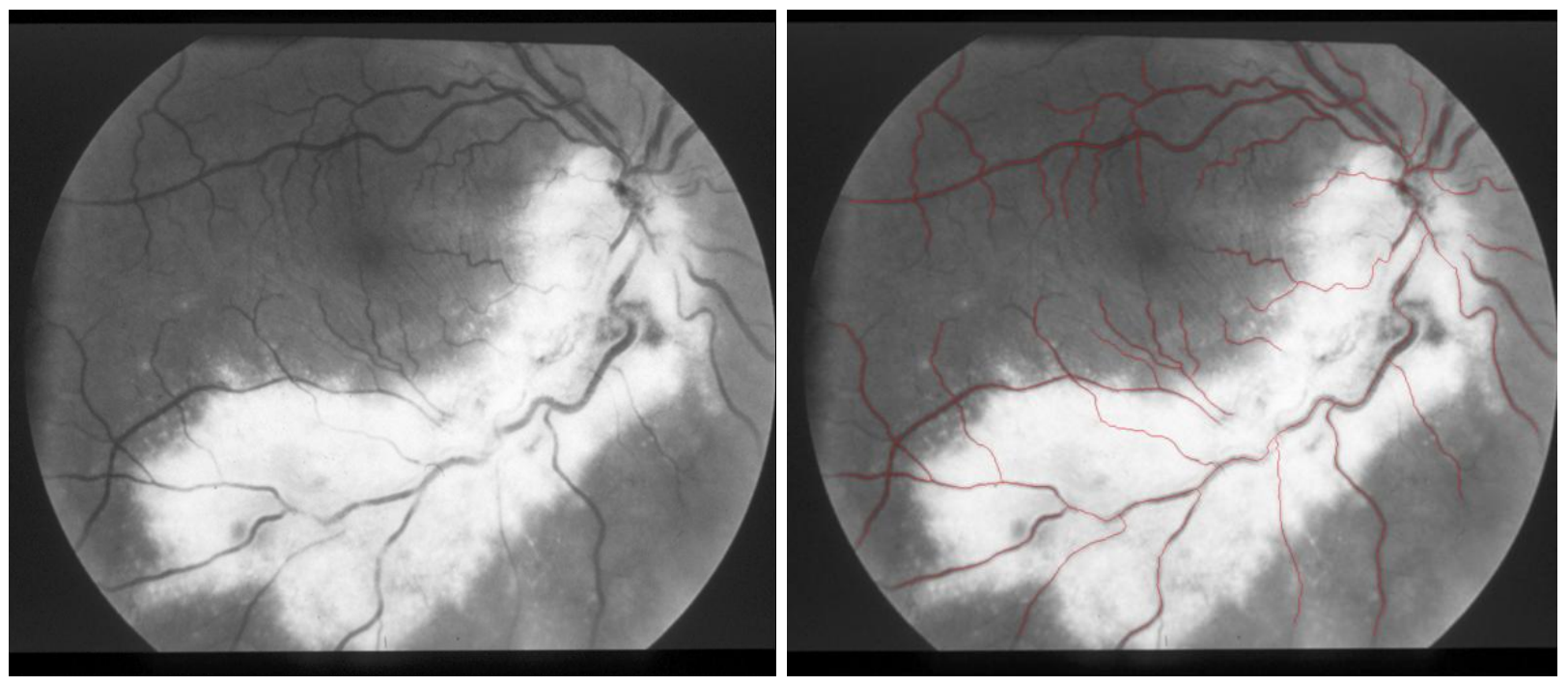

Figure 5. The result obtained for the retinal image. The original image is shown on the left and the centerlines found is shown on the right.
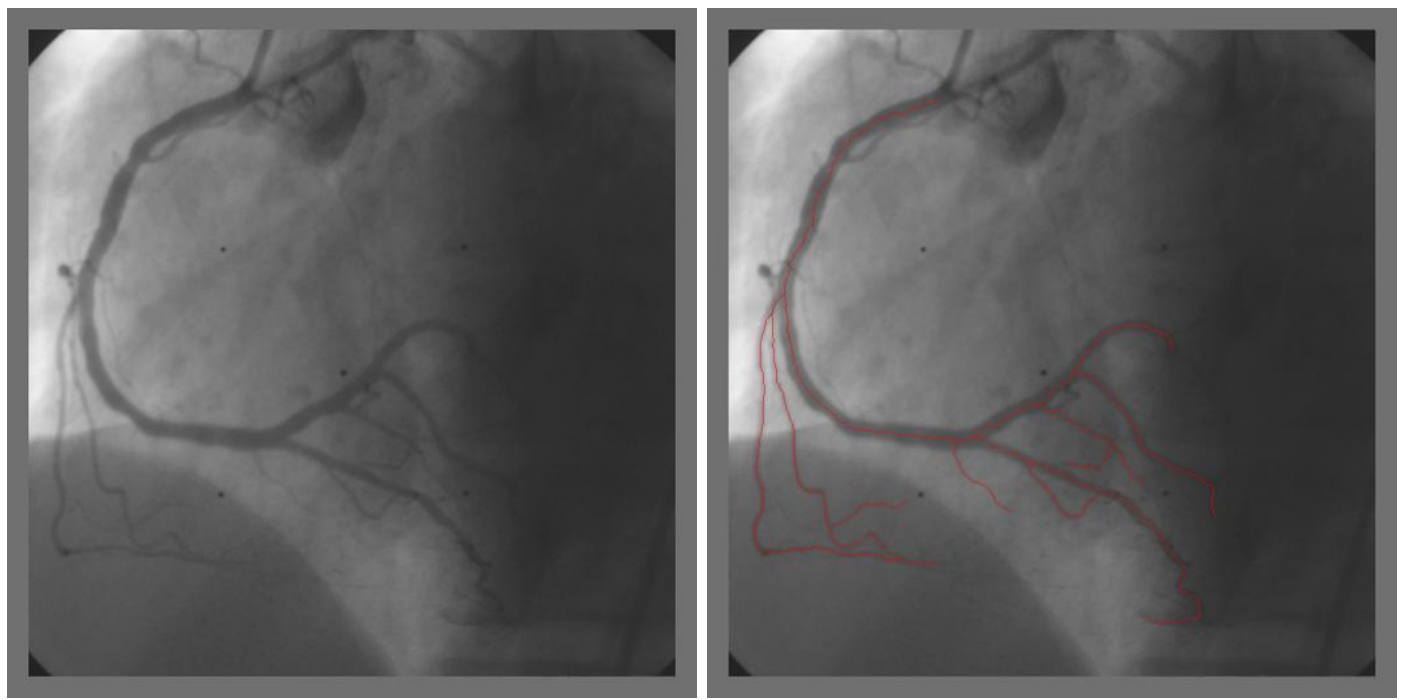

Figure 6. Center lines identification for the coronary artery in the angiogram. The image on the right shows the extracted centerlines. 

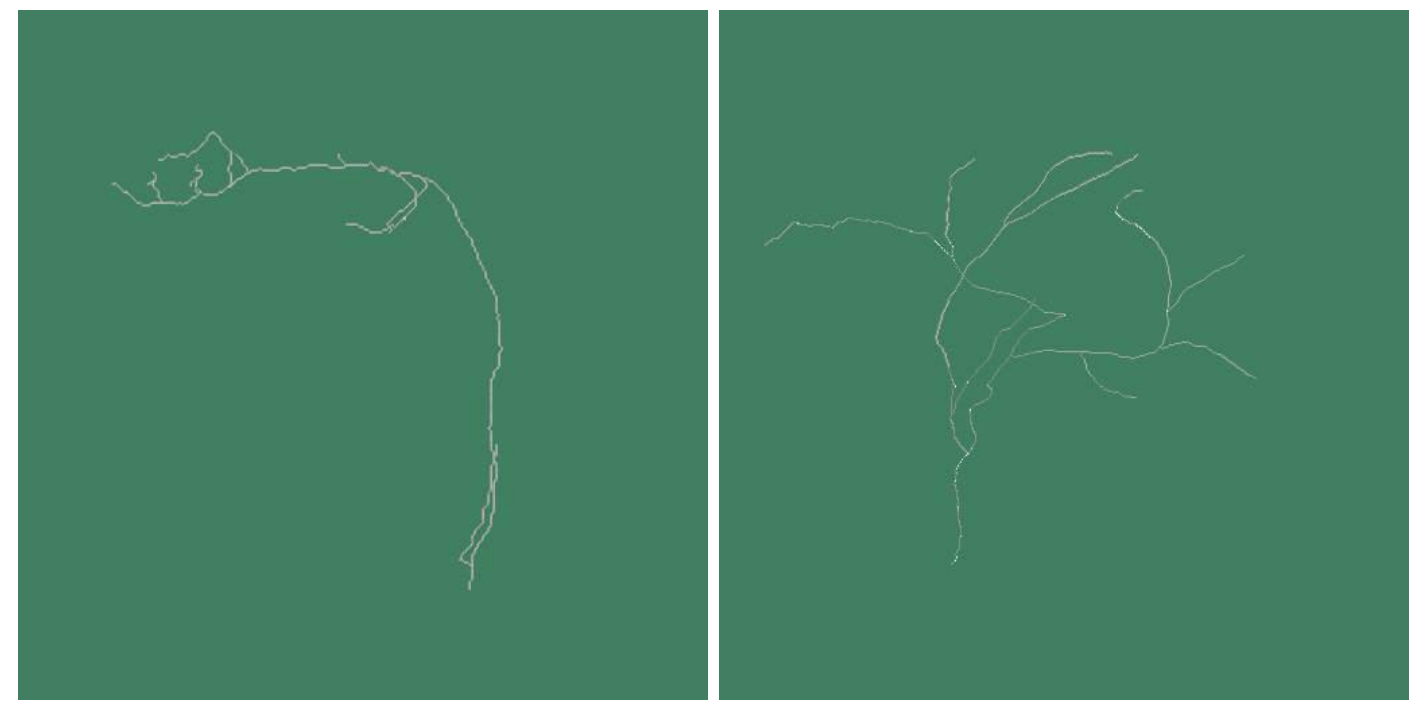

Figure 7. Two examples show the results of tracing neuron fibers. The images are 3D confocal microscopic images.

\section{CONCLUSION AND DISCUSSION}

We presented a semi-automatic method to find the centerline of tubular structure in 2D or 3D volume images. A problem with this approach is the long preprocessing time. However, the method worked well even for 3D neuron tracing case in confocal microscopic images. The future work will be segmentation of vessels or neuron fibers based on the centerline extracted.

\section{ACKNOWLEDGMENTS}

This work was supported under the grant NSC-92-2213-E009-100, National Science Council, Taiwan.

\section{REFERENCES}

1. D. Marr and D. Hildereth, "Theory of edge detection." Proc. Roy Soc, Ser. B, vol. 207, pp. 287-217, 1980

2. J. Canny, "A Computational Approach to Edge Detection," IEEE Trans. PAMI, vol. 8, pp. 679-697, 1986.

3. T. Elfving, J.O. Eklundh, and S. Nyberg, "Edge Detection Using the Marr-Hildreth operator with different sizes," Int. Conf. On Pattern Recognition, pp. 1109-1112, 1982.

4. W. Frei and C.C. Chen, "Fast boundary detection: A generalization and a new algorithm,," IEEE Trans. Comput., vol. 8, pp. 988-998, Oct. 1977.

5. F.M. Dickey, K.S. Shanmuugam, and J.A. Gree, "An optimal frequency domain filter fir edge detection in digital pictures," IEEE Trans. Anal, Machine Intell., pp. 37-49, Ian. 1979.

6. K.W. Mondestino and R.W. Fries, "Edge detection in noisy images using recursive digital filtering," Comput. Graphics and Image Processing, vol. 6, pp. 409-433, 1911.

7. V. Torre and T.A. Poggio, "One edge detection," IEEE PAMI., vol PAMI-8, pp. 147-163, 1979.

8. P. H. Eichel, E.J. Delp, K. Koral, and A. J. Buda, "A method for fully automatic definition of coronary arterial edges from cineagiograms," IEEE Trans. Med. Imaging, vol. 18, pp. 313-320, 1988.

9. K. Haris, S. N. Efstratiatiadis, N. Magkaveras, C. Pappas, J Gourassas, and G. Louridas, "Model based morphological Segmentation and labeling of coronary artery angiograms," IEEE Trans. Med. Imaging, vol. 18, pp. 1003-1015, 1999.

10. N. Ezquerra, S Capell, L. Klein, and P. Duijves, "Model-Guided Labeling of Coronary Structure," IEEE Trans. Med. Imaging, vol. 17, no. 3, jun. pp 429-441, 1998.

11. R. C. Chan, W. C. Karl, and R. S. Lees, "A New Model-Based Technique for Enhanced Small-Vessel Measurements in X-Ray Cine-Angiograms," IEEE Trans. Med. Imaging, vol. 19, pp. 243-255, Mar., 2000. 
12. Y. Sun, "Automated identification of vessel contours in coronary arteriograms by an adaptive tracking algorithm," IEEE Trans. Med. Imaging, vol. 8, pp. 78-88, Mar., 1989.

13. S. Tamura, K. Tanaka, S. Ohmori, K. Okazaki, A. Okada, and M. Hoshi, "Semiautomatic leakage analyzing system for time series fluorescein ocular fundus angiography," Pattern Reconition, vol. 16, no. 2, pp. 149-162, 1983.

14. Y. Tolias and S. Panas, "A fuzzy vessel tracking algorithm for retinal images based on fuzzy clustering," IEEE Trans. Med. Imaging, vol. 17, pp. 263-273, Apr., 1998.

15. S. Chaudhuri, S. Chatterjee, N. Katz, M. Nelson, and M. GOLDBAUM, "Detection of blood vessels in retinal images using two-dimensional matched filter," IEEE Trans. Med. Imaging, vol. 8, pp. 263-269, 1989.

16. A. Hoover, V. Kouznetsova, and M. Goldbaum, "Locating Blood Vessels in Retinal Images by Piecewise threshold probing of a matched filter response," IEEE Trans. Med. Imaging, vol. 8, pp. 203-210, 2000.

17. Y. Sun, R. J. Lucariello, and S. A. Chiaramida, "Directional Low-Pass Filtering for Improved Accuracy and Reproducibility of Stenosis Quantification in Coronary Arteriograms," IEEE Trans. Med. Imaging, vol. 14, No. 2, pp. 242-248, Jun.,2000.

18. T. Lindeberg, Scale-Space Theory in Computer Vision: Kluwer Academic Publishers, 1994.

19. T. Lindeberg, "Feature detection with automatic scale selection," International Journal of Computer Vision, vol. 30, pp. 79-116, 1998.

20. T. Lindeberg, "Edge detection and ridge detection with automatic scale selection," International Journal of Computer Vision, vol. 30, pp. 117-154, 1998.

21. Y. Sato, S. Nakajima, H. Atsumi, T. Koller, G. Gerig, S. Yoshida, and R. Kikinis, "3D multi-scale line filter for segmentation and visualization of curvilinear structures in medical images," Cvrmed-Mrcas'97, vol. 1205, pp. 213-222, 1997.

22. A. F. Frangi, W. J. Niessen, K. L. Vincken, and M. A. Viergever, "Multiscale vessel enhancement filtering," Medical Image Computing and Computer-Assisted Intervention - Miccai'98, vol. 1496, pp. 130-137, 1998.

23. O. Wink, A. F. Frangi, B. Verdonck, M. A. Viergever, and W. J. Niessen, "3D MRA coronary axis determination using a minimum cost path approach," Magnetic Resonance in Medicine, vol. 47, pp. 1169-1175, 2002.

24. O. Wink, W. J. Niessen, and M. A. Viergever, "Multiscale vessel tracking," Medical Imaging, IEEE Transactions on, vol. 23, pp. 130-133, 2004. 